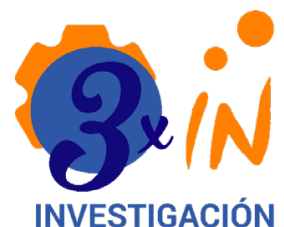

INVESTIGACIÓN E INNOVACION en INGENERIAS ISSN2344-8652

OPEN ACCESS

Recibido: 26/05/2021 Aceptado: 11/08/2021 Publicado: 13/09/2021

Correspondencia de autores: juane.sierra@udea.edu.co

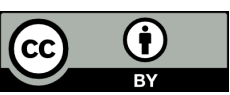

Copyrith 2020 by Investigación e Innovación en Ingenierías

\section{Metodología computacionalmente eficiente para resolver el despacho económico multiperiodo estocástico con restricciones de seguridad}

\section{Computationally efficient methodology to solve the stochastic security constrained unit commitment problem}

\author{
$\begin{array}{ll}\text { Jesús María López Lezama } & \text { JDan Sierra Aguilar } \\ \text { Cristian Camilo Marín Cano } & \text { iD Alvaro Jaramillo Duque iD }\end{array}$ \\ Universidad de Antioquia, Colombia
}

Resumen

Objetivo: Validar una metodología computacionalmente eficiente para resolver el problema de despacho económico multiperiodo estocástico con restricciones de seguridad. Metodología: Se utilizan factores lineales de sensibilidad para calcular flujos de carga de forma rápida y precisa. También se usa un método iterativo, que identifica y agrega como cortes de usuario, las restricciones de seguridad activas. Estas restricciones establecen la región factible de un modelo embebido dentro de un algoritmo Progressive Hedging, el cual descompone el problema principal en un conjunto de sub-problemas computacionalmente más tratables, al relajar las restricciones de acoplamiento entre escenarios. Resultados: Los resultados numéricos sobre el sistema IEEE RTS96, muestran que la estrategia propuesta entrega soluciones de alta calidad en bajos tiempos de cálculo. Conclusiones: La metodología propuesta permite solucionar el despacho económico multiperiodo estocástico seguro hasta 50 veces más rápido cuando se compara con su formulación extensiva.

Palabras clave: Despacho económico, restricciones de seguridad, factores lineales de sensibilidad, algoritmo Progressive Hedging.

Abstract

Objective: Validate a computationally efficient approach to solve the stochastic security constraint unit commitment problem. Methodology: Linear sensitivity factors are used to compute power flows in a fast and accurate way. It is also used an iterative method that identifies and adds as user cuts the set of active constraints. These constraints establish the feasible region of a model embedded within a Progressive Hedging algorithm, which decomposes the main problem into a set of computationally more tractable sub-problems by relaxing the coupling constraints between scenarios. Results: The numerical results on the IEEE RTS96 system show that the proposed strategy provides high quality solutions with low calculation times. Conclusions: The proposed methodology allows the stochastic security-constraint unit commitment problem to be solved up to 50 times faster when compared to its extensive formulation.

Keywords: Unit commitment, security constraints, linear sensitivity factors, Progressive Hedging algorithm.

Como citar (IEEE): J. Sierra-Aguilar., C. Marín-Cano., J. López-Lezama y A. Jaramillo-Duque “Metodología computacionalmente eficiente para resolver el despacho económico multiperiodo estocástico con restricciones de seguridad", Investigación e Innovación en Ingenierías, vol. 9, n², 130-141, 2021. DOI: https://doi.org/10.17081/invinno.9.2.4452 
Metodología computacionalmente eficiente para resolver el despacho económico multiperiodo estocástico con restricciones de seguridad

\section{Introducción}

El despacho económico multiperiodo con restricciones de seguridad (SCUC por sus siglas en inglés) es el proceso de optimización que se encarga de programar las unidades de generación del sistema de potencia, para suplir la demanda de energía, considerando los límites operacionales de las plantas de generación y las líneas de transmisión en operación normal y ante contingencias $\mathrm{N}-1$. El SCUC es un problema de programación entera mixta (MIP por sus siglas en inglés) y desde el punto de vista de su complejidad computacional es NP-completo [1]. La relajación Langrangiana (RL) y la descomposición de Benders (DB) han sido los métodos más explorados para solucionar el SCUC [2]. Uno de los factores que hace complejo el SCUC son sus restricciones de seguridad que aumentan la dimensión del problema. Para abordar este inconveniente, los autores en [3] proponen condiciones de factibilidad analítica para definir solamente el subconjunto de restricciones de seguridad activas del SCUC y en [4] se descartan matemáticamente las restricciones de seguridad innecesarias a través de condiciones necesarias y suficientes.

Actualmente, un factor importante en el desempeño computacional del SCUC es el uso de los factores lineales de sensibilidad. Estos son los Power Transfer Distribution Factors (PTDF) y Line Outage Distribution Factors (LODF), los cuales permiten calcular el flujo de las líneas ante cambios de inyección de potencia en barras y salida de operación de líneas (contingencias $\mathrm{N}-1$ ), respectivamente [5]. Actualmente se han desarrollado metodologías basadas en el uso de los PTDF y LODF para aumentar la eficiencia computacional de los modelos SCUC en sistemas de potencia a gran escala. Una forma de hacer esto es considerar solo restricciones vinculantes como se propone en [1] donde se utiliza la función de restricciones diferidas de Gurobi. En [6], se propone una metodología que agrega restricciones de seguridad necesarias como cortes de usuario, y que además permite calcular índices que suministran señales de expansión para la red de transmisión. Una forma alternativa de aumentar la eficiencia computacional del SCUC se basa en el concepto de cola de prioridades, agregando a lo sumo solo una restricción de seguridad N-1 por cada línea [7]. Estas metodologías tienen como objetivo principal generar de forma iterativa la región factible del problema SCUC, reduciendo considerablemente los tiempos de cómputo al evitar que la dimensión del problema aumente de forma innecesaria, Es importante destacar que hasta ahora ninguna de las técnicas anteriormente mencionadas ha sido aplicada en el análisis de la operación segura de sistemas eléctricos bajo condiciones de incertidumbre.

Recientemente, la evolución de los sistemas de potencia ha presentado grandes retos en la solución del SCUC debido a la incertidumbre de la integración de grandes cantidades de recursos renovables intermitentes [8,9]. La integración de este tipo de generación ha dado lugar al despacho económico multiperiodo estocástico con restricciones de seguridad (SSCUC por sus siglas en inglés). Este problema ha sido abordado usando escenarios e intervalos de optimización [10], descomposición de Benders [11] y programación estocástica multietapa [12]. Los anteriores trabajos evidencian la importancia y aplicación de los modelos SSCUC para abordar los desafíos de la incertidumbre sobre la operación segura de los sistemas eléctricos. En la solución del SSCUC la velocidad de cómputo es una característica de suma importancia para el operador de red al momento de tomar decisiones en tiempo real, dado que los modelos matemáticos del SSCUC son computacionalmente más complejos que su contra-parte determinística. Hoy en día existe poca literatura que aborde de manera estratégica la complejidad computacional del SSCUC. En este sentido, en [13] se propone un procedimiento totalmente paralelizado para resolver el SSCUC obteniendo soluciones eficientes y rápidas en sistemas eléctricos a gran escala y en [14] se propone un método que apunta a mejorar la eficiencia del SSCUC bajo optimización ordinal restricta. 
Siguiendo el enfoque de investigación abordado en [13,14], en este trabajo se propone una estrategia para resolver eficientemente el SSCUC considerando la incertidumbre de fuentes de generación intermitentes. La estrategia propuesta presenta las siguientes características: 1) Utiliza factores lineales de sensibilidad para calcular los flujos de potencia, reduciendo el número de variables y restricciones en comparación con el modelado de red convencional, 2) Incorpora cortes de usuario para agregar dinámicamente restricciones de seguridad activas como se propone en [6] y 3) utiliza el algoritmo de descomposición Progressive Hedging para relajar el modelo SSCUC. Las características anteriormente descritas permiten abordar el SSCUC de forma computacionalmente eficiente, reduciendo sustancialmente el tiempo de cálculo.

\section{Modelo Matemático}

El modelo matemático del SSCUC está basado en la formulación y modelamiento empleados en [15,16]. A diferencia de [15], la formulación de las restricciones de transmisión es realizada a través de PTDF para la operación normal y LODF para la operación bajo contingencias N-1. El modelo matemático propuesto está dado por las ecuaciones (1)-(10).

$$
\operatorname{Min} \sum_{\xi=1}^{g} p_{\xi} \cdot\left[\sum_{t=1}^{T} \sum_{i=1}^{I} C_{i}(t, \xi)+\sum_{t=1}^{T} \sum_{s=1}^{s} c^{s h} \cdot L_{s}^{s h}(t, \xi)+\sum_{t=1}^{T} \sum_{w=1}^{W} c_{\omega} \cdot Q_{w}(t, \xi)\right]
$$

Sujeto a:

$$
\text { Restricciones de estado de unidades y rampas }
$$

$$
\begin{gathered}
C_{i}(t, \xi)=a_{i} \cdot x_{i}(t)+\sum_{b=1}^{B} k_{i, b} \cdot g_{i, b}(t, \xi)+S U C_{i}(t) \forall t \leq T, i \leq I, \xi \leq \Xi \\
g_{i}(t, \xi)=\sum_{b=1}^{B} g_{i, b}(t, \xi) \forall t \leq T, i \leq I, \xi \leq \Xi \\
g_{i}(t, \xi) \geq g_{i}^{\min } \cdot x_{i}(t) \forall t \leq T, i \leq I, \xi \leq \Xi \\
g_{i, b}(t, \xi) \leq g_{i}^{\max } \cdot x_{i}(t) \forall t \leq T, i \leq I, b \leq B, \xi \leq \Xi \\
-F_{l}^{\max } \cdot T C F \leq \sum_{s} \frac{P_{T D F}}{l, s} \cdot P_{s}^{N e t}(t, \xi) \leq F_{l}^{\max } \cdot T C F \forall l \leq L, t \leq T, \xi \leq \Xi \\
-F_{l}^{\max } \cdot T C F \leq F_{l}(t, \xi)+\overline{L O D F}_{l, k} \cdot F_{k}(t, \xi) \leq F_{l}^{\max } \cdot T C F \forall l \leq L, k \leq K, t \leq T, \xi \leq \Xi \\
0 \leq L_{s}^{s h}(t, \xi) \leq D_{s}(t) \forall s \leq S, t \leq T, \xi \leq \Xi \\
0 \leq Q_{w}(t, \xi) \leq g_{w}(t, \xi) \forall w \leq W, t \leq T, \xi \leq \Xi
\end{gathered}
$$


La función objetivo indicada en la ecuación (1) consiste en minimizar el valor esperado del costo total de operación del sistema; el cual está dado por los costos de la generación de las plantas térmicas $C_{i}(t, \xi)$ , los costos asociados con el deslastre de carga $L_{s}^{s h}(t, \xi)$, y los costos asociados con el deslastre de potencia eólica $Q_{w}(t, \xi)$. En este caso, los índices $t_{\mathrm{y}} \xi$ corresponden al periodo de tiempo y al escenario, respectivamente.

Por simplicidad, las restricciones de estado de los generadores y de rampas se han indicado forma general en la expresión (2). Estas restricciones son típicas en los modelos de despacho óptimo multiperiodo y pueden ser consultadas en detalle en [16]. La restricción dada por la ecuación (3) corresponde al costo de operación de las plantas térmicas. En este caso, $a_{i}$ es el costo de producción fijo de la planta i, $x_{i}(t)$ es una variable de decisión binaria (variable de estado) que indica si la planta i ha sido programada en el periodo $\mathrm{t}$; $k_{i, b}$ es el costo de producción variable del generador i para el tramo b (se consideran costos lineales a tramos), $g_{i, b}(t, \xi)$ indica la notencia entregada por el generador térmico i, en el segmento b en el intervalo de tiempo t en el escenario ${ }^{\xi}$; finalmente, $S U C_{i}(t)$ es el costo asociado al arranque de la unidad i en el periodo de tiempo t. La restricción (4) define la potencia total del generador térmico i expresada como la suma de los niveles de generación de la curva de costo. Las restricciones (5) y (6) indican los límites mínimos y máximos de la potencia entregada por los generadores. Note que estas restricciones son multiplicadas por la variable de estado del generador. Las restricciones (7) y (8) indican los límites de flujo de potencia en operación normal y bajo contingencia, respectivamente. En este caso, $F_{l}^{\max }{ }_{\text {indica el límite }}$ térmico de la línea l, $T C F$ es el factor de capacidad de transmisión, $\overline{P T D F}_{l, s}$ es el factor de distribución de transferencia de potencia asociado a la línea l cuando hay una inyección en el nodo s, $\overline{L O D F}_{l, k}$ es el factor de distribución (porcentaje de carga) que asume la línea l ante una falla en la línea $\mathrm{k}$ y $F_{k}(t, \xi)$ es el flujo en la línea $k$ en el periodo $t$ en el escenario $\xi$. Las restricciones (8) y (9) representan el límite de deslastre de carga y de generación eólica. En este caso, $D_{s}(t)$ y $g_{w}(t, \xi)$ son la demanda del nodo s en intervalo de tiempo t y el pronóstico de generación eólica en el intervalo de tiempo t en el escenario $\xi$, respectivamente.

\section{Metodología}

El modelo descrito por las ecuaciones (1)-(10) presenta el fenómeno de explosión combinatorial dadas las posibles combinaciones de los estados (on-off) de los generadores, las contingencias $\mathrm{N}-1$ de las líneas y los escenarios de operación. A continuación se describe la estrategia de solución propuesta.

\section{Metodología para adicionar restricciones $\mathrm{N}-1$}

Por medio de los LODF es posible modelar contingencias $\mathrm{N}-1$ con bajo costo computacional como se indica en $[1,5,6]$. Matemática y computacionalmente no tiene sentido considerar todas las posibles contingencias en las líneas transmisión; puesto que no siempre se generan sobrecargas en otras líneas de transmisión del sistema. Por lo tanto, para tener en cuenta solo las restricciones vinculantes que definen la región factible del problema, se implementó la metodología propuesta en [6], la cual está basada en el concepto de cortes de usuario. Estos cortes son restricciones lineales agregadas de manera estratégica por el usuario, basado en la información implícita del problema. El Algoritmo 1 muestra la metodología propuesta por [6] para adicionar, como cortes de usuario, las restricciones $\mathrm{N}-1$ suficientes y necesarias para el modelo. 
Algoritmo 1. Metodología para añadir las restricciones de seguridad N-1

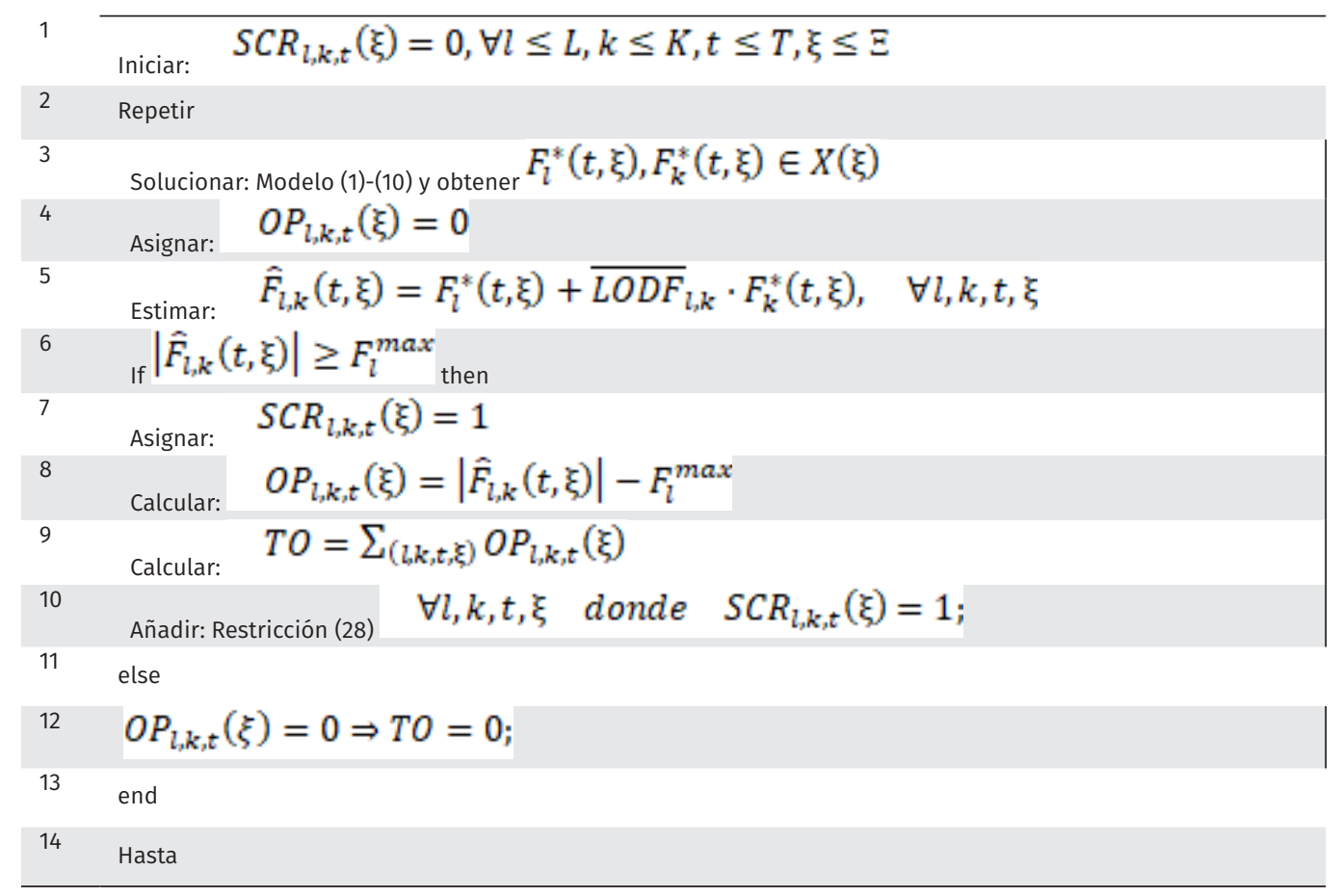

\section{Fuente: Elaboración propia}

En este caso, $S C R_{l, k, t}(\xi)$ es un arreglo binario de cuatro dimensiones que toma el valor 1 si hay una sobrecarga en la línea l, para la contingencia k, en el periodo de tiempo t, en el escenario $\xi$. Análogamente, $O P_{l, k, t}(\xi)$ es un arreglo binario de cuatro dimensiones que almacena el valor de la sobrecarga de la línea 1 ruandn nrurre la contingencia k, en el periodo de tiempo t, en el escenario $\xi$. En el paso 1 del Algoritmo 1 , $S C R_{l, k, t}(\xi)$ se inicia en cero, para considerar solamente el caso base, sin tener en cuenta las restricciones de seguridad N-1. A partir de un proceso repetitivo (paso 2), el modelo definido por las ecuaciones (1)-(10) es solucionado de manera iterativa (paso 3). Dentro de este proceso, desde el paso 4 hasta el paso 9 , se estiman las restricciones de seguridad N-1 directamente vinculadas con la región factible de problema SSCUC. En el paso 4, el parámetro $O P_{l, k, t}(\xi)$ debe ser reiniciado en cero, con el objetivo de almacenar, posteriormente, el valor de sobrecarga únicamente de nuevas restricciones de seguridad N-1. En paso 5, el parámetro $F_{l, k}^{\wedge}(t, \xi)$ estima los flujos post-contingencia de las líneas con base en los flujos óptimos

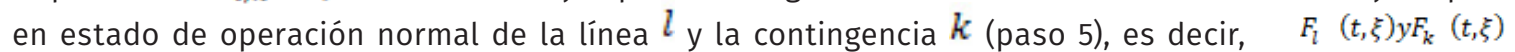
, respectivamente, los cuales son previamente calculados en paso 3. Estimado el valor de $\widehat{F_{1, k}}(t, \xi)$ y considerando la máxima capacidad de transmisión para cada línea $F_{1}^{\max }$, es posible calcular y almacenar los valores de las sobrecargas generadas en las líneas a través del parámetro $\mathrm{OP}_{1, \mathrm{k}, \mathrm{t}}(\xi)$; adicionalmente, este parámetro permite calcular la sobrecarga total del sistema $T O$, que se utiliza para definir la condición de parada del algoritmo. En el paso 10 se realiza la activación u adición de las restricciones de seguridad $\mathrm{N}-1$, para las combinaciones de $\mathrm{l}, \mathrm{k}, \mathrm{t}, \xi$ donde $\operatorname{SCR}_{1, k, t}(\xi)$ sea igual a 1; este último parámetro permite registrar y contar todas las sobrecargas en cada iteración. En el paso 12, si no existen sobrecargas desde la primera iteración del algoritmo entonces $\mathrm{OP}_{1, k, \mathrm{t}}(\xi)$ es cero y por lo tanto $\mathrm{TO}=0$. El paso 14 es el final del proceso iterativo cuando $T O$ vale cero o es menor a un valor de tolerancia tol (muy pequeño), para este caso tol $=1^{-7} \mathrm{MW}$. Para una descripción más detallada de esta metodología, el lector interesado puede referirse a [6]. 


\section{Algoritmo Progressive Hedging (PHA)}

En términos generales, la forma extensiva de un problema de optimización estocástico consiste en un único modelo matemático donde todas las restricciones son escritas para todos los escenarios [17]. Si se trata de un problema a gran escala, esta formulación no garantiza su solución por falta de recursos computacionales. El PHA es un método de descomposición basado en escenarios, propuesto inicialmente en [18], donde el problema se soluciona de forma independiente por cada escenario, al relajar la restricción de no anticipatividad, permitiendo obtener buenas soluciones con alta eficiencia computacional [19]. Además, el PHA ha demostrado ser mucho más estable que otros algoritmos de amplio uso en la literatura como la DB [17]. Por estas razones, el PHA ha sido recientemente utilizado, como un método promisorio, para solucionar problemas de la operación estocástica de sistemas eléctricos, tal como la programación y despacho de generación y la planeación hidrotérmica de corto y mediano plazo [20].

El Algoritmo 2 ilustra de forma compacta el funcionamiento del PHA. Una descripción detallada del mismo está fuera del alcance de este documento, pero puede ser consultada en [19]. En este caso $\rho$ es un escalar o vector de la misma longitud de la variable no anticipativa $x$; en el paso 1, se inicia el contador de iteración $v=0$ y el vector de pesos $w^{0}(\xi)=0$, el cual tiene la misma dimensión de ${ }^{x}$. En los pasos 2-4, la solución inicial de los sub-problemas del escenario es obtenida. En el paso 5 se actualiza la iteración ${ }^{v}$. En el paso 6 , el algoritmo define su mejor estimación actual de una solución no anticipatividad, es decir, $\bar{x}^{v}$ no depende de la realización del escenario $\xi$. En el paso 7, se estiman los multiplicadores $w(\xi)$ necesarios para actualizar la condición de no anticipatividad. En los pasos 8-10, los multiplicadores $w(\xi)$ son usados junto con un término proximal al cuadrado con el objetivo de encontrar una solución óptima donde los valores $x$ no dependan de los escenarios.

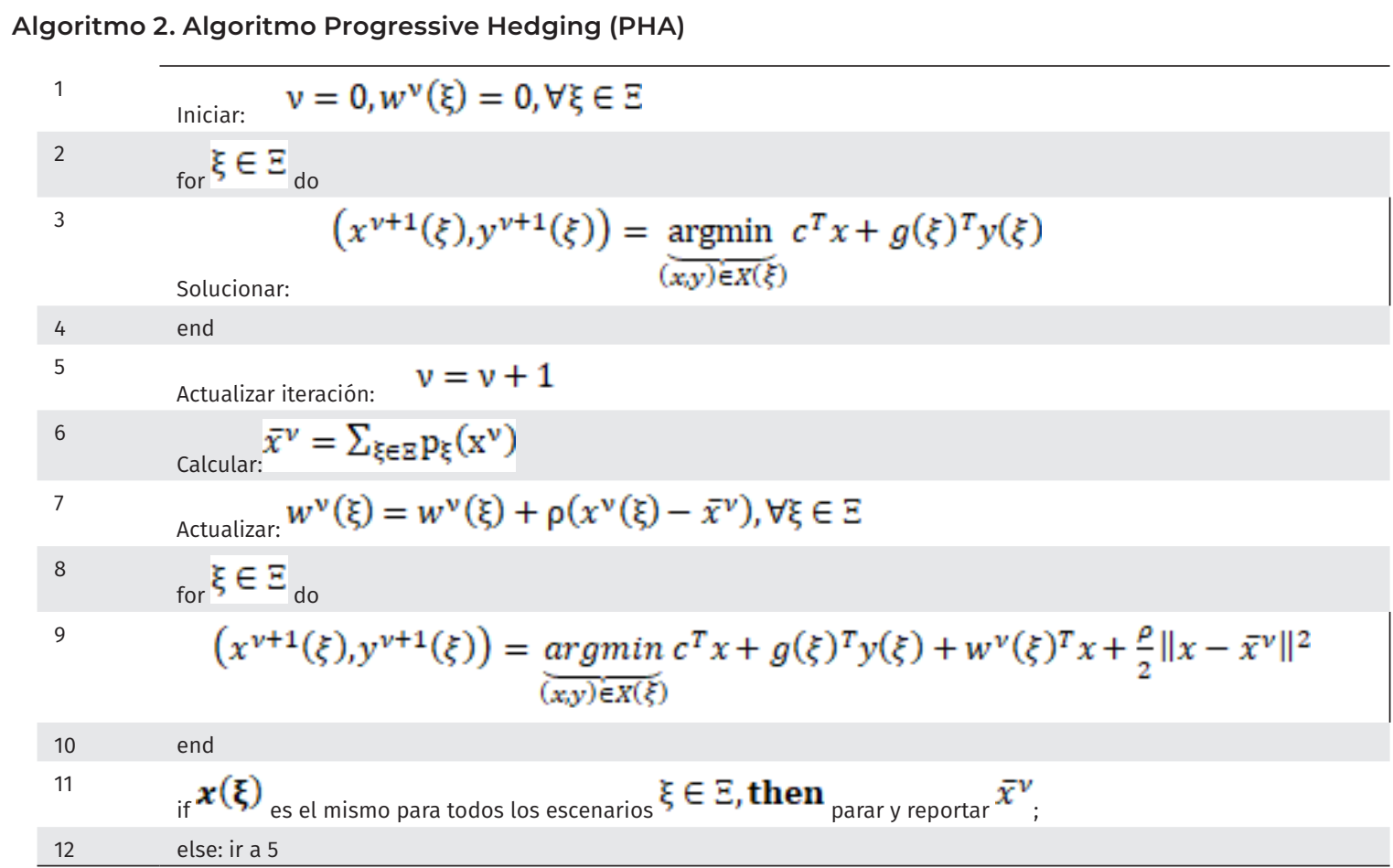

Fuente: Elaboración propia 


\section{Integración de restricciones de seguridad $\mathrm{N}-1$ en el PHA}

El Algoritmo 3 muestra la inserción de la metodología iterativa de adicionar restricciones de seguridad $\mathrm{N}-1$ dentro del PHA. En los pasos 1-2 se ajustan e inicializan los parámetros de trabajo necesarios para la metodología de adicción de restricciones N-1 y para el PHA, respectivamente. En los pasos 3-4 se actualiza la iteración e inicializa el proceso iterativo del PHA donde se resuelven los sub-problemas de cada escenario. Los pasos 5-17 definen la metodología de adicción de restricciones N-1 propuesta, la cual se encuentra embebida dentro del proceso iterativo de PHA; donde $x^{v}(\xi)$ es la variable de cobertura del PHA en cada iteración $v$ y en cada escenario $\xi$. En este caso, la variable de cobertura es la variable binaria $x \in\{0,1\}$ que asigna el estado on/off de cada unidad de generación. Una vez encontrada una solución a través de la metodología de adicción de cortes $\mathrm{N}-1$, los pasos 18-21 son la continuación del PHA para encontrar una solución óptima donde $x^{v}(\xi)$ sea igual en cada escenario (condición no anticipatividad).

Algoritmo 3. Metodología para añadir restricciones N-1 dentro del PHA

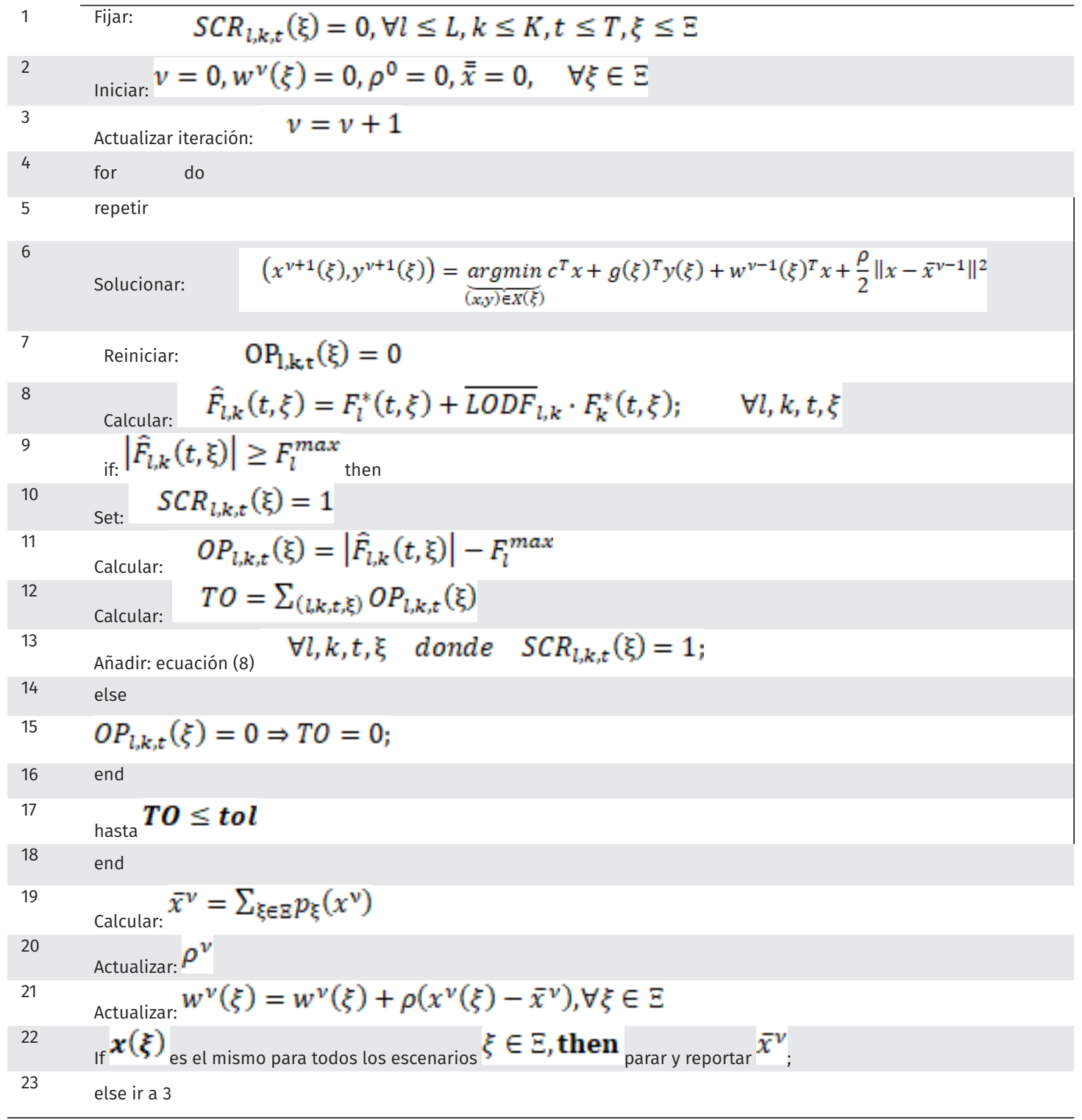

Fuente: Elaboración propia 


\section{Resultados}

La estrategia computacional propuesta en este artículo fue probada en dos equipos computo: una estación de cálculo con procesadores Intel Xeon E5 @ 2,40GHz de 44 núcleos y una memoria RAM de 256 GB, y un computador de escritorio Intel Core i7 @ 3.4GHz de 8 núcleos con una memoria RAM de 8 GB. El sistema de prueba es el IEEE RTS-96 también usado en [16], que se ilustra en la figura 1, con un horizonte de tiempo de 24 horas y cuya información está totalmente disponible en [21]. El sistema está compuesto por 73 barras, 120 líneas de transmisión, 96 generadores térmicos y 19 granjas eólicas (con una capacidad instalada de 6900MW y localizadas en las barras 102, 114, 116, 118, 121, 119, 123, 202, 212, 213, 219, 220, 223, 301, 306, 309; también tiene 51 cargas con una demanda máxima de 7539 MW y demanda promedio de 6258 MW. La solución del modelo matemático del problema SCUC N-1 para este sistema, considerando 10 diferentes escenarios cada uno con su probabilidad, fue obtenida utilizando el software comercial de modelamiento algebraico GAMS versión 24.8.5, bajo el solver CPLEX.

Figura 1. Ilustración del sistema de prueba.

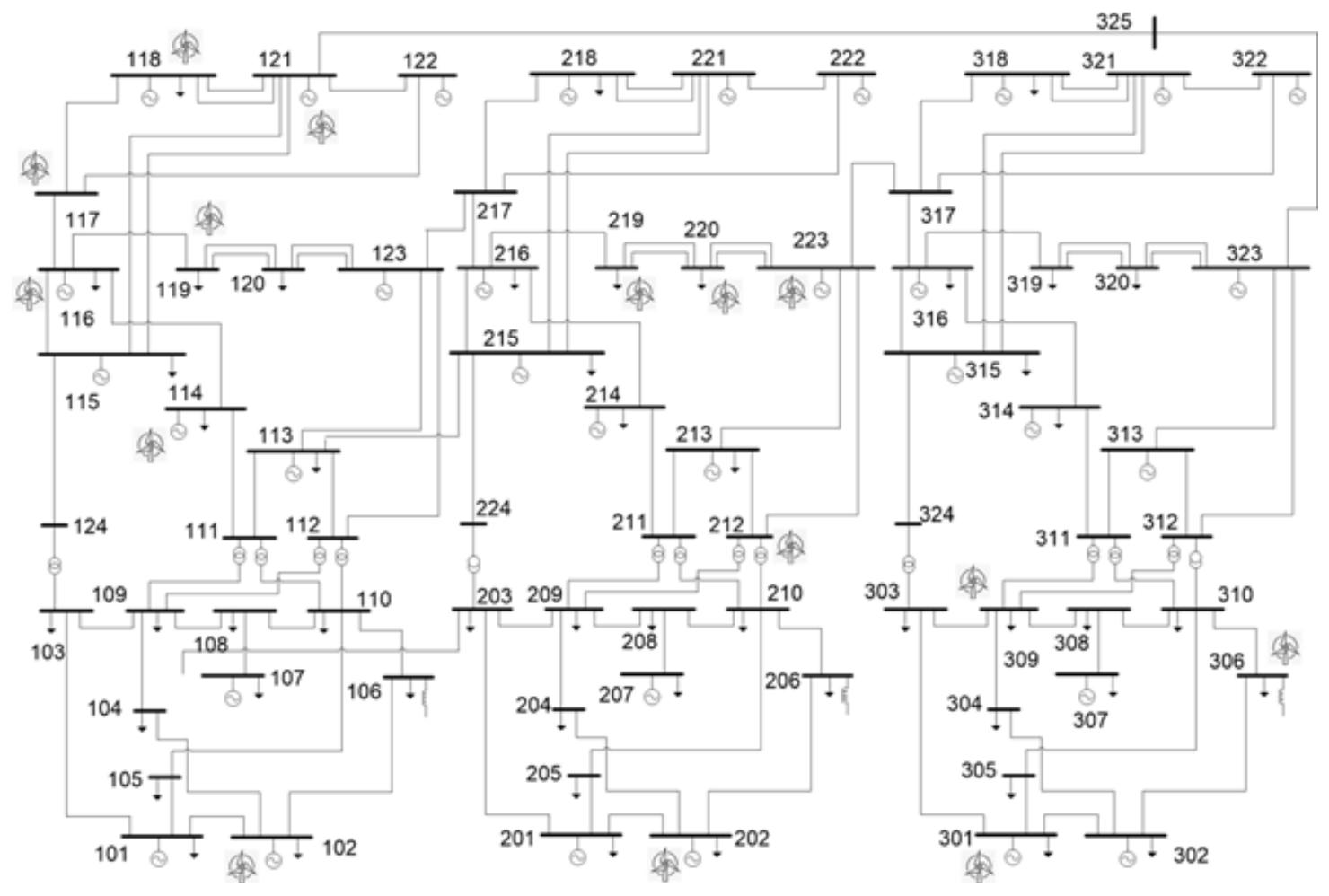

Fuente: Elaboración propia

\section{Ajuste de parámetros del PHA}

Uno de los primeros parámetros a ser ajustado es el factor de penalización $\rho$. Teniendo en cuenta que los valores de $\rho$ con base en costos de producción de energía de la variable de cobertura son los más comunes, dado su buen desempeño en la convergencia del PHA [22], la estrategia propuesta en [23] para la actualización de $\rho$ fue implementada. Otro factor importante es la selección de la variable de cobertura. Algunos autores han realizado una serie de experimentos para observar cual es la mejor variable de cobertura dentro del problema UC [22, 24]. Bajo diferentes estrategias de ajuste de $\rho$, en [22] se ilustra que la variable de cobertura con mejor desempeño es la variable de estado on/off de los generadores; esta variable fue la adoptada para las simulaciones. 
Finalmente, para garantizar convergencia y soluciones factibles del PHA, usando el método de redondeo, se seleccionaron valores de los parámetros $\alpha_{\mathrm{y}} \beta$, para diferentes pruebas de la estrategia computacional propuesta. Durante las pruebas realizadas en la estación de cálculo, los valores de $\beta$ y $\alpha$ fueron 0 y 0,53, respectivamente, mientras que en las pruebas realizadas en el el computador de escritorio los valores fueron 0 y 0,57 , respectivamente.

\section{Análisis y comparación de resultados}

Para validar el desempeño de la estrategia propuesta en este trabajo, se realizó un análisis comparativo con la formulación extensiva del SSCUC. La formulación extensiva del SSCUC se utiliza para conocer el gap de optimalidad, y el valor de la solución óptima (la mejor solución relajada) del problema. El gap de optimalidad mínimo definido en el software GAMS, para la solución del SSCUC en ambas formulaciones, fue de $1 \%$. En este caso, las soluciones encontradas serán de alta calidad si este gap es cercano al $1 \%$.

Para considerar la incertidumbre de la generación eólica se consideraron 10 escenarios descritos en [16]. En la Tabla 1 se presentan los resultados obtenidos. En este caso, se compara la función objetivo (costo esperado del despacho), el tiempo de solución, y el gap de optimalidad, obtenidos en la estación de cálculo y el computador de escritorio para ambas formulaciones. Note que la formulación extensiva solamente pudo ser solucionada usando la estación de cálculo, pues debido a la dimensión del problema, el computador de escritorio no contaba con la suficiente memoria y recursos computacionales para resolverla. Esto se debe a que en la formulación extensiva del SSCUC las 118 líneas en contingencia N-1 generan 3,3 millones de restricciones adicionales; sin embargo, solo 116 mi restricciones de seguridad N-1 vinculantes son necesarias para definir la región factible, descartando casi el $96 \%$ de las restricciones usadas en la formulación extensiva.

En la estación de cálculo, la formulación extensiva del SSCUC toma 61222 segundos (aproximadamente 17 horas) para alcanzar un valor un gap de 1,1\%, obteniendo una función objetivo de 1,1110 MUSD. Por otro lado, la formulación propuesta, al ser un modelo de dimensión más compacto es solucionada en apenas 1194 segundos en la estación de cálculo y 1564 segundos cuando se usa el computador de escritorio. En ambos casos se tienen gaps de optimalidad de 1,62 y 2,12\%, respectivamente. Se puede observar que los tiempos de solución de la formulación propuesta son de un orden de magnitud aproximadamente 50 veces menor que la formulación extensiva del modelo. Si bien la formulación propuesta presenta un gap de optimalidad mayor que la formulación extensiva, esto es compensado por un cálculo mucho más veloz, lo cual es uno de los principales requerimientos de los operadores de red para solucionar el SSCUC. Usando la formulación y estrategia de solución propuesta el operador de red tiene la flexibilidad de calcular soluciones aceptables del SSCUC en tiempos de cálculo rápido que ayudan a coordinar acciones operativas útiles en la seguridad y confiabilidad de la red, pero sacrificando parte de la economía del sistema.

\section{Tabla 1. Comparación de resultados}

\begin{tabular}{cccc}
\hline Formulación & Costo [MUS\$̦] & Tiempo [s] & Gap [\%] \\
\hline Extensiva (estación de cálculo) & 1,1110 & 61222 & 1,10 \\
\hline Propuesta (estación de cálculo) & 1,1167 & 1194 & 1,62 \\
\hline Propuesta (computador de escritorio) & 1,1223 & 1564 & 2,12 \\
\hline
\end{tabular}

Fuente: Elaboración propia 


\section{Conclusiones}

El despacho económico mulitperiodo estocástico con restricciones de seguridad (SSCUC por sus siglas en inglés), es uno de los problemas más desafiantes en la operación de los sistemas eléctricos. En este artículo se presentó una formulación y estrategia de solución alternativa al SSCUC. La principal ventaja de la metodología propuesta radica en la velocidad de cálculo. Esto se logra mediante una estrategia conjunta que utiliza los factores de sensibilidad lineales PTDF y LODF para un cálculo rápido de las inyecciones de potencia y los flujos post-contingencia, respectivamente. Además, se utiliza una técnica novedosa (cortes de usuario) que solamente considera las restricciones vinculantes al problema debido a contingencias $\mathrm{N}-1$. Finalmente, ambas estrategias se implementan dentro del algoritmo Progressive Hedging para considerar la estocasticidad de la generación eólica mediante escenarios. Los resultados en el sistema de prueba IEEE RTS96 permiten concluir que la metodología propuesta es efectiva y eficaz para solucionar el SSCUC. Las estrategias de reducción de tiempo de cómputo permiten encontrar soluciones de alta calidad de un orden de magnitud 50 veces más rápido que la formulación convencional del SSCUC. Contar con metodologías computacionalmente eficientes para solucionar el SSCUC es de vital importancia para los operadores de red, pues los tiempos de cálculo pueden llegar a ser inviables para aplicaciones reales. En este sentido la metodología propuesta ofrece una alternativa atractiva entre velocidad de cálculo y calidad de las soluciones encontradas.

A pesar de que el PHA es más estable que otros algoritmos de descomposición por etapas como la descomposición de Benders, es recomendable probar el desempeño de la metodología de adición de restricciones de seguridad N-1 con este tipo de técnicas, incluso investigar su desempeño bajo la fusión del PHA y otros métodos de descomposición prometedores como Frank Wolfe o descomposición de Fenchel, que no han sido trabajados en el análisis del SSCUC.

\section{Referencias bibliográficas}

1. D. A. Tejada-Arango, P. Sánchez-Martın, y A. Ramos, “Security Constrained Unit Commitment Using Line Outage Distribution Factors", IEEE Transactions on Power Systems, vol. 33, núm. 1, pp. 329-337, ene. 2018. DOI: https://doi.org/10.1109/TPWRS.2017.2686701.

2. R. Lu, T. Ding, B. Qin, J. Ma, X. Fang, y Z. Dong, “Multi-Stage Stochastic Programming to Joint Economic Dispatch for Energy and Reserve With Uncertain Renewable Energy", IEEE Transactions on Sustainable Energy, vol. 11, núm. 3, pp. 1140-1151, jul. 2020. DOI: https://doi.org/10.1109/TSTE.2019.2918269.

3. H. Wu, X. Guan, Q. Zhai, y H. Ye, "A Systematic Method for Constructing Feasible Solution to SCUC Problem With Analytical Feasibility Conditions", IEEE Transactions on Power Systems, vol. 27, núm. 1, pp. 526-534, feb. 2012. DOI: https://doi.org/10.1109/TPWRS.2011.2165087.

4. A. J. Ardakani y F. Bouffard, "Identification of umbrella constraints in DC-based security-constrained optimal power flow", en 2014 IEEE PES General Meeting Itextbar Conference \& Exposition, jul. 2014, pp. 1-6.

5. Allen J. Wood, Bruce F. Wollenberg, y Gerald B. Sheble, Power Generation, Operation, and Control., 3rd ed. Wiley, 2013.

6. C. C. Marín-Cano, J. E. Sierra-Aguilar, J. M. López-Lezama, Á. Jaramillo-Duque, y W. M. Villa-Acevedo, "Implementation of User Cuts and Linear Sensitivity Factors to Improve the Computational Performance of the Security-Constrained Unit Commitment Problem", Energies, vol. 12, núm. 7, p. 1399, ene. 2019. DOI: https://doi.org/10.3390/en12071399 
7. Á. S. Xavier, F. Qiu, F. Wang, y P. R. Thimmapuram, “Transmission Constraint Filtering in Large-Scale Security-Constrained Unit Commitment", IEEE Transactions on Power Systems, vol. 34, núm. 3, pp. 2457-2460, may 2019. DOI: https://doi.org/10.1109/TPWRS.2019.2892620

8. T. G. Hlalele, R. M. Naidoo, J. Zhang, y R. C. Bansal, "Dynamic Economic Dispatch With Maximal Renewable Penetration Under Renewable Obligation", IEEE Access, vol. 8, pp. 38794-38808, 2020, doi: https://doi.org/10.1109/ACCESS.2020.2975674.

9. P. Shinde, M. R. Hesamzadeh, P. Date, y D. W. Bunn, "Optimal Dispatch in a Balancing Market with Intermittent Renewable Generation", IEEE Transactions on Power Systems, pp. 1-1, 2020. DOI: https:// doi.org/10.1109/TPWRS.2020.3014515.

10. L. Wu, M. Shahidehpour, y Z. Li, “Comparison of Scenario-Based and Interval Optimization Approaches to Stochastic SCUC", IEEE Transactions on Power Systems, vol. 27, núm. 2, pp. 913-921, may 2012. DOI: https://doi.org/10.1109/TPWRS.2011.2164947

11. O. Alizadeh-Mousavi y M. Nick, "Stochastic Security Constrained Unit Commitment with variablespeed pumped-storage Hydropower Plants", en 2016 Power Systems Computation Conference (PSCC), jun. 2016, pp. 1-7.

12. H. Park, Y. G. Jin, y J.-K. Park, "Stochastic security-constrained unit commitment with wind power generation based on dynamic line rating", International Journal of Electrical Power \& Energy Systems, vol. 102, pp. 211-222, nov. 2018. DOI: https://doi.org/10.1016/j.ijepes.2018.04.026

13. C. Wang y Y. Fu, "Fully Parallel Stochastic Security-Constrained Unit Commitment", IEEE Transactions on Power Systems, vol. 31, núm. 5, pp. 3561-3571, sep. 2016. DOl: https://doi.org/10.1109/ TPWRS.2015.2494590

14. N. Yang, D. Ye, Z. Zhou, Y. Huang, y B. Dong, "Research on Solving Method of Security Constrained Unit Commitment Based on Improved Stochastic Constrained Ordinal Optimization", en 2018 3rd International Conference on Intelligent Green Building and Smart Grid (IGBSG), abr. 2018, pp. 1-4.

15. H. Pandžić, Ting Qiu, y D. S. Kirschen, "Comparison of state-of-the-art transmission constrained unit commitment formulations", en 2013 IEEE Power \& Energy Society General Meeting, jul. 2013, pp. 1-5.

16. H. Pandzic, Y. Dvorkin, T. Qiu, Y. Wang, y D. Kirschen, Unit Commitment under Uncertainty - GAMS Models. University of Washington.

17. S. Ryan, R. J.-B. Wets, D. Woodruff, C. Silva-Monroy, y J.-P. Watson, Toward scalable, parallel progressive hedging for stochastic unit commitment, en Proceedings of the 2013 IEEE Power Energy Society General

18. Meeting, Vancouver, BC, Canada, 21-25 July 2013; pp. 1-5.

19. R. T. Rockafellar y R. J.-B. Wets, "Scenarios and Policy Aggregation in Optimization Under Uncertainty", Math. Oper. Res., vol. 16, núm. 1, pp. 119-147, feb. 1991. DOI: https://doi.org/10.1287/moor.16.1.119.

20. D. Gade, G. Hackebeil, S. M. Ryan, J.-P. Watson, R. J.-B. Wets, y D. L. Woodruff, "Obtaining lower bounds from the progressive hedging algorithm for stochastic mixed-integer programs", Mathematical Programming, vol. 157, núm. 1, pp. 47-67, may 2016. DOI: https://doi.org/10.1007/s10107-016-1000-z

21. R. E. C. Gonçalves, E. C. Finardi, y E. L. da Silva, "Applying different decomposition schemes using the progressive hedging algorithm to the operation planning problem of a hydrothermal system", Electric Power Systems Research, vol. 83, núm. 1, pp. 19-27, feb. 2012. DOI: https://doi.org/10.1016/j. epsr.2011.09.006

22. WashU_. University of Washington, 2018. 
23. C. Ordoudis, P. Pinson, M. Zugno, y J. M. Morales, "Stochastic unit commitment via Progressive Hedging; extensive analysis of solution methods", en 2015 IEEE Eindhoven PowerTech, jun. 2015, pp. 1-6.

24. J.-P. Watson y D. L. Woodruff, "Progressive hedging innovations for a class of stochastic mixed-integer resource allocation problems", Computational Management Science, vol. 8, núm. 4, pp. 355-370, nov. 2011. DOI: https://doi.org/10.1007/s10287-010-0125-4

25. Li Chao, Zhang Muhong, y Hedman Kory W., "N-1 Reliable Unit Commitment via Progressive Hedging", Journal of Energy Engineering, vol. 141, núm. 1, p. B4014004, mar. 2015. DOI: https://doi.org/10.1061/ (ASCE)EY.1943-7897.0000187 